\title{
Comparative studies of placentation and immunology in non-human primates suggest a scenario for the evolution of deep trophoblast invasion and an explanation for human pregnancy disorders
}

\author{
Anthony M Carter \\ Cardiovascular and Renal Research, Institute of Molecular Medicine, University of Southern Denmark, \\ J. B. Winsloewsvej 21, DK-5000 Odense, Denmark
}

Correspondence should be addressed to A M Carter; Email: acarter@health.sdu.dk

\begin{abstract}
Deep trophoblast invasion in the placental bed has been considered the hallmark of human pregnancy. It occurs by two routes, interstitial and endovascular, and results in transformation of the walls of the spiral arteries as they traverse the decidua and the inner third of the myometrium. Disturbances in this process are associated with reproductive disorders such preeclampsia. In contrast, trophoblast invasion in Old World monkeys occurs only by the endovascular route and seldom reaches the myometrium. Recently, it was shown that this pattern is maintained in gibbons, but that the human arrangement also occurs in chimpanzee and gorilla. There is an interesting parallel with results from placental immunology regarding the evolution of the major histocompatability complex class I antigen HLA-C and its cognate receptors. HLA-C is not present in Old World monkeys or gibbons. It emerged in the orangutan and became polymorphic in the lineage leading to gorilla, bonobo, chimpanzee, and human. Interaction between HLA-C1 and HLA-C2 on the surface of trophoblast and killer immunoglobulin-like receptors (KIRs) expressed by uterine natural killer cells are important regulators of trophoblast invasion. Evolution of this system in great apes may have been one prerequisite for deep trophoblast invasion but seems to have come at a price. The evidence now suggests that certain combinations of maternal genotype for KIRs and fetal genotype for HLA-C imply an increased risk of preeclampsia, fetal growth restriction, and recurrent abortion. The fetal genotype is in part derived from the father providing an explanation for the paternal contribution to reproductive disorders.

Reproduction (2011) 141 391-396
\end{abstract}

\section{Introduction}

Preeclampsia is a disease that affects about $4 \%$ of human pregnancies and is a major cause of neonatal and maternal morbidity and mortality (Ilekis et al. 2007). A key feature of the disease, to which symptoms such as hypertension, proteinuria, and edema can be attributed, is a generalized dysfunction of the maternal vascular endothelium (Roberts \& Lain 2002). Proteinuria, for example, is due to characteristic changes in the renal glomeruli (glomerular endotheliosis). Indeed, a strict diagnosis of preeclampsia, differentiating it from other forms of pregnancy hypertension, requires a renal biopsy (Chesley 1980) or demonstration of podocytes in the urine (Garovic et al. 2007).

Advances in our understanding of the symptoms of this disease have brought us no nearer a clarification of its etiology. It is, however, recognized that many pregnancy disorders, including fetal growth restriction and preeclampsia, are associated with defective placentation. In healthy pregnancies, extravillous trophoblast invades the uterine wall through the entire depth of the endometrium and the inner third of the myometrium. This process is associated with complete remodeling of the walls of the spiral arteries, leading to gross widening of the vessel lumen and ensuring an adequate flow of blood to the intervillous space (Pijnenborg et al. 2006, Harris 2011). In pregnancy disorders, trophoblast invasion is shallower, never reaching the myometrium, and transformation of the spiral arteries is less extensive. Exactly how this defect in placental circulation is connected to the endothelial disorder in preeclampsia continues to be researched and debated.

It is often stated, albeit incorrectly, that preeclampsia is a uniquely human disease (e.g. Robillard et al. 2002). The idea has arisen that a high rate of blood flow in late gestation was required to enable fetal brain development. This was achieved through the evolution of deeper trophoblast invasion than, for example, in Old World monkeys. It may have come at a price, namely the occurrence of preeclampsia in pregnancies where 
trophoblast invasion was shallow. The theory is best articulated by Martin (2007), although he is careful to argue that deep invasion is just one solution to the problem as whales and dolphins have large brains at birth yet an entirely different type of placentation.

A number of factors control the rate and extent of trophoblast invasion in primates, but the focus of this review is on recent advances in reproductive immunology. There is an interaction between, on the one hand, major histocompatability complex (MHC) class I molecules expressed by the extravillous trophoblast and, on the other, a family of receptors expressed by a unique population of immune cells, the uterine natural killer cells (uNK cells; Moffett \& Hiby 2007). The co-evolution of these molecules has been worked out in an elegant set of studies in non-human primates. It can be shown to parallel the evolution of trophoblast invasion revealed by our own studies of the placental bed in gibbons, chimpanzees, and gorillas. What makes this story so interesting is the recent demonstration (Hiby et al. 2010) that a high expression of one MHC class I molecule on trophoblast (HLA-C2) is associated with an increased risk of pregnancy disorders such as preeclampsia. This is a gene dosage effect that occurs when the allele is inherited from the father. The observation thus offers an explanation for the paternal contribution to the incidence of preeclampsia that is well known from epidemiological studies (llekis et al. 2007).

\section{Trophoblast invasion in human pregnancy}

Trophoblast is derived from the trophectoderm of the blastocyst and is the only fetal tissue presented to the mother. It clothes the terminal villi of the placenta and is the primary route of maternal-fetal exchange. The villi are the outermost branches of villous trees that descend from the chorionic plate and attach to the basal plate by anchoring villi. The trophoblast that invades the uterine wall and spiral arteries is referred to as extravillous trophoblast. It invades by two routes (Pijnenborg et al. 2006). The interstitial route pertains to trophoblast that invades from the anchoring villi into the underlying decidua and the inner third of the myometrium. Invasion also occurs by the endovascular route as trophoblast migrates from the basal plate through the lumen of the spiral arteries.

Transformation of the spiral arteries occurs in two phases. The first comprises endothelial swelling and a loss in coherence of the vascular smooth muscle. It occurs before the appearance of endovascular trophoblast and is possibly mediated by factors secreted by uNK cells (Harris 2011). In the second phase, the vessel endothelium is lost, and the vascular smooth muscle and elastic fibers are further broken down, while fibrinoid material is deposited in the vessel wall. This phase is associated with incorporation into the vessel wall of trophoblast that has invaded by both interstitial and endovascular routes. The net effect of all these changes is a great increase in diameter of the vessels supplying blood to the intervillous space.

In healthy pregnancies, these changes occur in the segments of arteries that pass through both the inner third of the myometrium and the full depth of the decidua. In preeclampsia and some other pregnancy disorders, the extravillous trophoblast does not reach the myometrium. The corresponding arterial segments therefore retain their endothelium and smooth muscle and are not dilated (Pijnenborg et al. 2006).

\section{Trophoblast invasion in non-human primates}

There are two important differences between Old World monkeys and humans. First, little or no trophoblast invasion occurs by the interstitial route. Instead, there is a sharp border at the fetal-maternal interface between a cytotrophoblastic shell and the decidua (Fig. 1). The shell
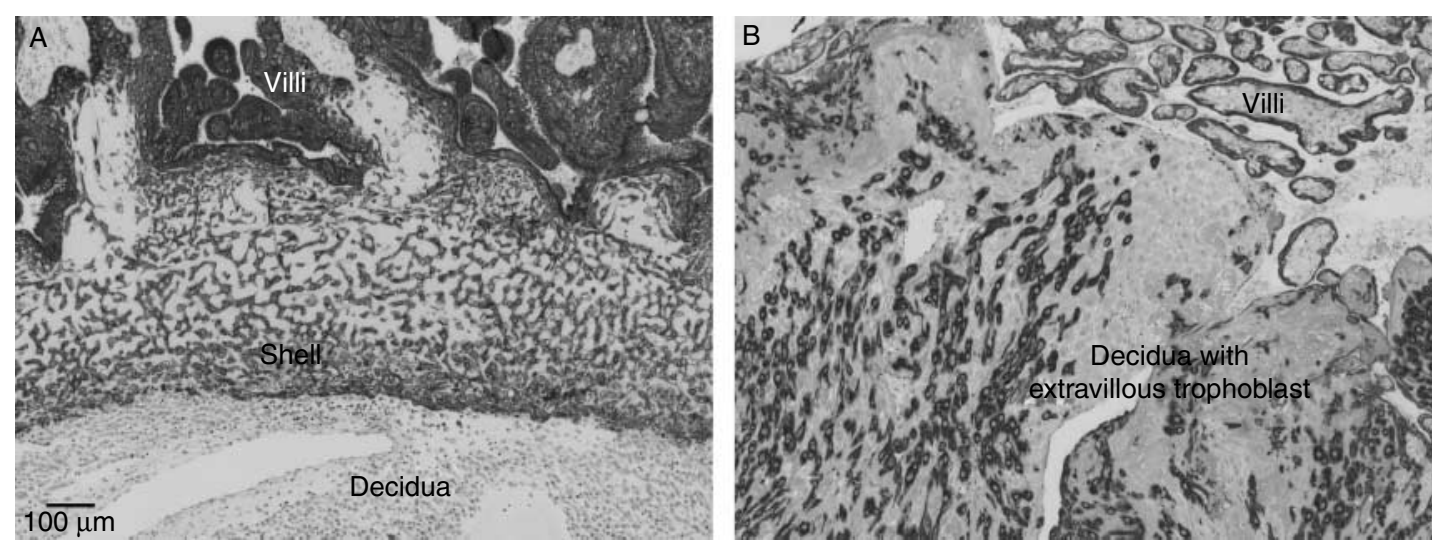

Figure 1 Comparison between the fetal-maternal interface in the baboon and the human. Tissue sections were immunostained for cytokeratin to identify trophoblast. (A) In the baboon, there is a sharp border between the cytotrophoblastic shell and the underlying decidua. (B) In the human placental bed, extravillous trophoblast invades by the interstitial route; it reaches deep into the decidua (shown) and progresses into the inner myometrium. Reproduced with permission from Pijnenborg et al. (2010) (C) 2010 Cambridge University Press. 
consists of sedentary trophoblast cells embedded in extracellular matrix and remains largely intact throughout pregnancy (Pijnenborg et al. 1996, Enders \& Blankenship 1997). Secondly, although there is early and rapid invasion of trophoblast by the endovascular route (Enders et al. 1996), it does not penetrate as deep. Reports differ as to whether it reaches the myometrium in the rhesus macaque (Ramsey et al. 1976, Blankenship et al. 1993). It does not reach the myometrial segments of the spiral arteries in the baboon; therefore, these segments do not normally undergo transformation (Pijnenborg et al. 1996).

These findings fit quite well with the idea that deep trophoblast invasion was evolved in humans as an aid to fetal brain development (Martin 2007). Until recently, it was not known whether this feature was unique to humans or occurred in our close relatives the great apes. Recently, we were able to address this question using archival specimens of ape uteri from museum collections (Carter \& Martin 2010, Carter \& Pijnenborg 2011).

The superfamily Hominoidea comprises two families. The family Hylobatidae includes the lesser apes or gibbons. They have a cytotrophoblastic shell as Old World monkeys (Carter \& Pijnenborg 2011, Pijnenborg et al. 2010). The family Hominidae includes orangutans, gorillas, bonobos, chimpanzees, and human beings. Interestingly, the pattern of trophoblast invasion in gorilla and chimpanzee pregnancies resembles the human condition (Fig. 1). There is invasion by the interstitial and endovascular routes, it extends to the inner third of the myometrium, and consequently there is transformation of the myometrial segments of the spiral arteries (Carter \& Martin 2010).

It will shortly be observed that these resemblances parallel the evolution of the immune system in primates. What are the implications for preeclampsia? It is commonly stated, often with Chez (1976) as the authority, that preeclampsia is a uniquely human disease. However, there are several case reports of eclampsia in gorilla and chimpanzee (Stout \& Lemon 1969, Baird 1981, Thornton \& Onwude 1992). In one instance, a renal biopsy was taken, and the distinctive features associated with human preeclampsia were shown (Stout \& Lemon 1969).

\section{Interaction between extravillous trophoblast and uNK cells in human pregnancy}

As already observed, extravillous trophoblast invades the decidua and blood vessels by the interstitial and endovascular routes. It is not solely responsible for transformation of the uterine spiral arteries. Early events in this process include focal loss of the vessel endothelium and disruption of the vascular smooth muscle. These changes are coincident with the influx of uNK cells (Harris 2011). Although they are derived from the same lineage, uNK cells have different properties from the natural killer cells found in the blood and elsewhere (Lash et al. 2010). They are a source of angiogenic growth factors and matrix-degrading proteases and therefore able to initiate changes in the spiral arteries (Harris 2011). Later in the process of spiral artery transformation, there is crosstalk between uNK cells and extravillous trophoblast. This centers around the interaction between HLA-C molecules expressed at the surface of trophoblasts and their cognate receptors found on the uNK cells.

MHC class I molecules play a key role in cellular immunity. HLA-A and HLA-B, which are highly polymorphic, are not expressed at the cell surface by extravillous trophoblasts. Instead, these cells express HLA-E, HLA-G, and HLA-C (Hunt et al. 2005). HLA-E is the product of an ancient gene, but HLA-G - which is expressed only by trophoblast - and HLA-C are much more recent (Older Aguilar et al. 2010). Importantly, HLA-E and HLA-G are invariant, whereas HLA-C exists in two variants, HLA-C1 and HLA-C2. The latter, therefore, are the only class I molecules on trophoblast with the potential to act as fetal allogens.

MHC class I molecules act as ligands for an extraordinary number of killer immunoglobulin-like receptors (KIRs; Moffett \& Hiby 2007, Abi-Rached et al. 2010a). These may be activating or inhibitory in nature. For present purposes, it is sufficient to observe that, in humans, expansion of lineage III of the KIR genes has yielded KIR2DL2/3, an inhibiting receptor specific for the C1 epitope, and KIR2DL1, an inhibiting receptor for the C2 epitope. In addition, there is an activating receptor for the C2 epitope named KIR2DS1. The corresponding genes are in a locus within the leukocyte receptor complex on chromosome 19. In the uterus, these receptors are expressed by uNK cells, which make up $70 \%$ of the leukocyte population. The depth of trophoblast invasion in the uterus is thought to be controlled in large part through an interaction between KIR receptors expressed on uNK cells and the respective ligands expressed on the extravillous trophoblast.

A further complication, with implications for reproductive disorders, is the existence of two haplotypes for the KIR genes. Haplotype A has only the inhibitory receptors, whereas haplotype $B$ has both stimulating and inhibitory receptors (Moffett \& Hiby 2007). There are thus three genotypes: $\mathrm{AA}, \mathrm{AB}$, and $\mathrm{BB}$. The complete system includes receptors that recognize HLA-E (CD94:NKG2A) and HLA-G (KIR2DL4).

\section{HLA-C ligands and KIRs in non-human primates}

uNK cells are by no means confined to human pregnancy. As an example, they constitute the main lymphocyte population in the endometrium of the rhesus macaque (Slukvin et al. 2001). This raises the question of what KIR receptors they express and how they might 
Table 1 Evolution of trophoblast invasion compared with that of the major histocompatability complex class I antigen HLA-C in Old World monkeys, gibbons and hominids.

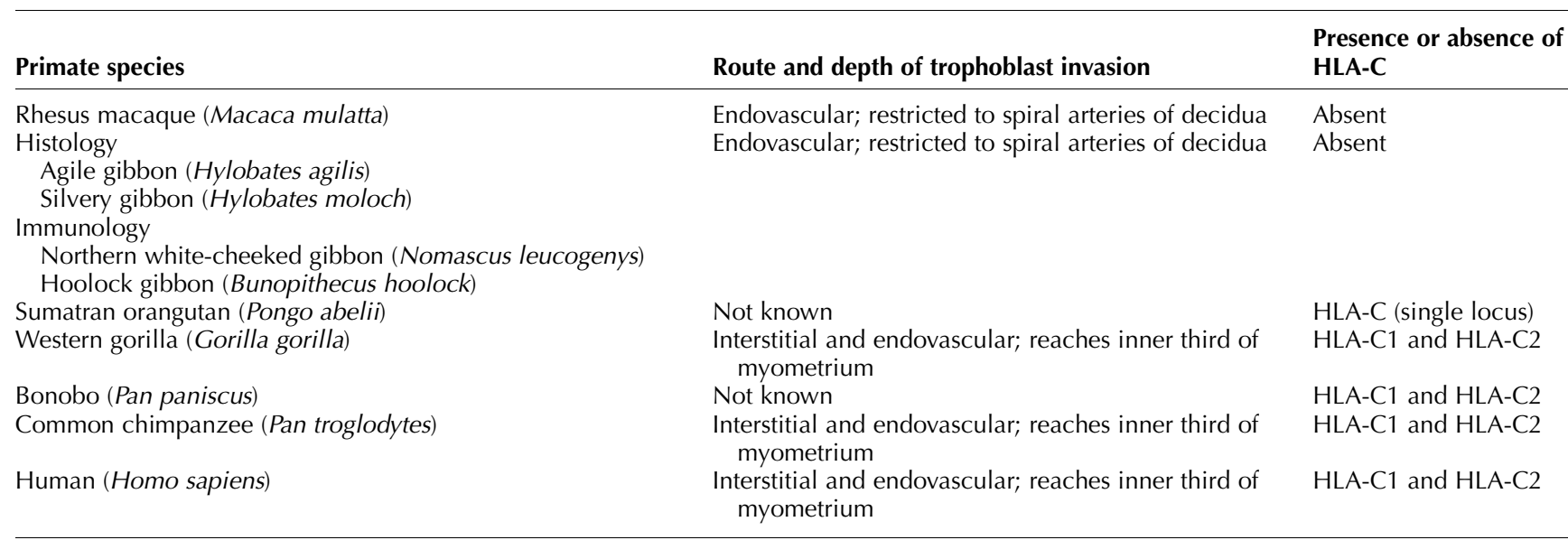

interact with ligands expressed on the surface of extravillous trophoblast.

HLA-C1 and HLA-C2 ligands are shared by chimpanzee and human, although there are some interesting differences in their cognate receptors (Abi-Rached et al. 2010b). In addition, both HLA-C variants are present in gorilla (Lawlor et al. 1991) and bonobo (Cooper et al. 1998). One might expect gibbons (the lesser apes) to be intermediate between Old World monkeys and the great apes, but a recent study (Abi-Rached et al. 2010a) has shown that their MHC class I ligands to have pursued quite a different course of evolution. Gibbons were found to lack HLA-C and HLA-G and to have quite different KIRs. Whereas in great apes partial or complete gene duplications have led to diversification of KIR haplotypes, in gibbons KIR haplotype diversity has been generated mainly through deletion events. As an example, expansion of lineage III KIR genes occurred in great apes, but this lineage was virtually extinguished in gibbons. Thus, in all likelihood, gibbons diverged from other hominoids before MHC-C arose (Abi-Rached et al. 2010a).

A recent study in the orangutan has offered a compelling scenario for the co-evolution in primates of MHC-C epitopes and their receptors (Older Aguilar et al. 2010). C1 and C1-specific KIRs evolved first and are already present in orangutans. Orangutans lack C2 and C2-specific KIRs. They do, however, have a novel KIR named Popy2DLB, which in the laboratory binds equally to $\mathrm{C} 1$ and $\mathrm{C} 2$. For the orangutan, which lacks C2, Popy2DLB can function only as a $\mathrm{C} 1$ receptor, but it has the potential to engage $\mathrm{C} 2$ should it arise. Consequently, Popy2DLB has the requisite properties of an evolutionary intermediate that would allow C2-mediated regulation to evolve from $\mathrm{C} 1$-mediated regulation without loss of function (Older Aguilar et al. 2010).

It is possible that co-evolution of MHC-C ligands and their cognate receptors played a role in the evolution of deep trophoblast invasion in gorillas, chimpanzees, and humans. Therefore, it is of note that trophoblast invasion in gibbons resembles that in Old World monkeys and is unlike that in chimpanzees or humans (Table 1). At present, we have no knowledge of the extent of trophoblast invasion in orangutans (Carter \& Pijnenborg 2011), and it is important to determine this should placental bed specimens ever become available.

\section{Implication of HLA-C ligands in pregnancy disease}

As observed above, there are two haplotypes for the human KIR receptors and thus three genotypes $A A, A B$, and $\mathrm{BB}$. In women with the AA genotype, the UNK cells express inhibitory receptors for the $\mathrm{C} 1$ and $\mathrm{C} 2$ epitopes but lack the activating receptor for C2 (Moffett \& Hiby 2007). During pregnancy, this predisposes to a higher incidence of preeclampsia (Hiby et al. 2004). Because trophoblast expresses both maternally and paternally inherited HLA-C molecules, Moffett \& Hiby (2007) hypothesized that there might be an increased risk of preeclampsia when the father contributed an HLA-C2

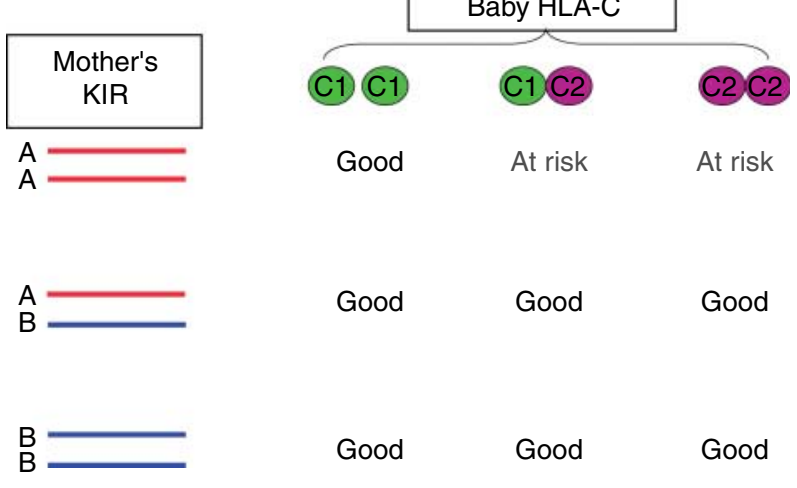

Figure 2 Certain combinations of maternal KIRs and fetal HLA-C genotypes increase the risk of a poor clinical outcome of pregnancy. Reproduced with permission from Moffett \& Hiby (2007) (c) 2007 Elsevier, Inc. 
allele (Fig. 2). This would result in trophoblast that expressed more HLA-C2 than did the maternal tissues. Later this hypothesis was extended to explain a paternal contribution to recurrent miscarriage (Hiby et al. 2008).

A new study (Hiby et al. 2010) has elaborated on previous work concerning interactions between HLA-C epitopes and their receptors. Importantly, genotyping for C1/C2 and KIRs was performed on maternal blood and on cord blood or mouth swabs from the babies of three cohorts of pregnant women including one with normal pregnancies. In a fourth cohort with recurrent miscarriage, blood samples were taken from the women and their partners. The findings supported the original hypothesis (Moffett \& Hiby 2007) and showed that C2 genes of paternal origin were associated with an increased risk of preeclampsia, fetal growth restriction, and recurrent miscarriage (Hiby et al. 2010). When the trophoblast has two $\mathrm{C} 2$ alleles (one paternally derived) but the mother has $\mathrm{C} 1 / \mathrm{C} 1$ or $\mathrm{C} 1 / \mathrm{C} 2$, there is a higher incidence of pregnancy disorders. There is thus a gene dosage effect. The extra C2 allele is paternally derived neatly solving the question of the known but hitherto unexplained paternal contribution to reproductive disorders (and suggesting that men with the $\mathrm{C} 2$ alleles might not be good sperm donors).

\section{Conclusion}

In summary, the patterns of trophoblast invasion revealed in our studies of non-human primates closely parallel the evolution of the HLA-C/KIR complex (Table 1). Thus, we have shown that there is deep invasion in chimpanzee and gorilla as in human pregnancy but not in gibbons or Old World monkeys. HLA-C is not present in the latter two groups. It emerged in the orangutan and became polymorphic in the lineage leading to gorilla, bonobo, chimpanzee, and human. This work on non-human primates demonstrates the potential inherent in comparative studies across species. It underlines the importance of responsible research on non-human primates and calls into question current legislation in the European Union (Abbott 2010) and United States (Cohen 2007, Ledford 2010) that severely hampers research on the great apes.

What remains unclear is the evolutionary advantage gained by duplication of the HLA-C gene. If there is a causal relationship between this event and the development of deep trophoblast invasion, as Parham et al. suggest (Abi-Rached et al. 2010a), this apparently comes at the cost of an added risk of reproductive disorders. When the chimpanzee genome was published, it was immediately noticed that the genes for KIRs had undergone rapid evolutionary divergence between chimpanzee and human (Varki \& Altheide 2005). These differences in the cognate receptors have now been studied in detail (Moesta et al. 2009, Abi-Rached et al. 2010b). Comparative studies show that human beings are the only hominid species without a C1-specific activating receptor (Moesta et al. 2010). While it is beyond doubt that preeclampsia can occur in the chimpanzee (Stout \& Lemon 1969), it has largely been overlooked. An intriguing possibility is that the incidence of the disease is indeed lower in chimpanzees because they have evolved a set of KIRs that minimizes the risk.

\section{Declaration of interest}

The authors declare that there is no conflict of interest that could be perceived as prejudicing the impartiality of the review reported.

\section{Funding}

This review did not receive any specific grant from any funding agency in the public, commercial or not-for-profit sector.

\section{References}

Abbott A 2010 Lab-animal battle reaches truce. Biomedical scientists say revised European directive on animal welfare averts feared disaster for research. Nature 464 964. (doi:10.1038/464964a)

Abi-Rached L, Kuhl H, Roos C, ten Hallers B, Zhu B, Carbone L, de Jong PJ, Mootnick AR, Knaust F, Reinhardt $\mathbf{R}$ et al. 2010a A small, variable, and irregular killer cell Ig-like receptor locus accompanies the absence of MHC-C and MHC-G in gibbons. Journal of Immunology $\mathbf{1 8 4}$ 1379-1391. (doi:10.4049/jimmunol.0903016)

Abi-Rached L, Moesta AK, Rajalingam R, Guethlein LA \& Parham P $2010 b$ Human-specific evolution and adaptation led to major qualitative differences in the variable receptors of human and chimpanzee natural killer cells. PLoS Genetics 6 e1001192. (doi:10.1371/journal.pgen. 1001192)

Baird JN Jr 1981 Eclampsia in a lowland gorilla. American Journal of Obstetrics and Gynecology 141 345-346.

Blankenship TN, Enders AC \& King BF 1993 Trophoblastic invasion and the development of uteroplacental arteries in the macaque: immunohistochemical localization of cytokeratins, desmin, type IV collagen, laminin, and fibronectin. Cell and Tissue Research 272 227-236. (doi:10.1007/ BF00302728)

Carter AM \& Martin RD 2010 Comparative anatomy and placental evolution. In Placental Bed Disorders, pp 85-96. Eds R Pijnenborg, I Brosens \& R Romero. Cambridge, UK: Cambridge University Press.

Carter AM \& Pijnenborg R 2011 Evolution of invasive placentation with special reference to non-human primates. Best Practice \& Research. Clinical Obstetrics \& Gynaecology [in press]. (doi:10.1016/j.bpobgyn. 2010.10.010)

Chesley LC 1980 Hypertension in pregnancy: definitions, familial factor, and remote prognosis. Kidney International 18 234-240. (doi:10.1038/ ki.1980.131)

Chez RA 1976 Nonhuman primate models of toxemia of pregnancy. In Hypertension in Pregnancy, pp 421-424. Eds MD Lindheimer, AI Katz\& FP Zuspan. New York: John Wiley \& Sons.

Cohen J 2007 Biomedical research. The endangered lab chimp. Science 315 450-452. (doi:10.1126/science.315.5811.450)

Cooper S, Adams EJ, Wells RS, Walker CM \& Parham P 1998 A major histocompatibility complex class I allele shared by two species of chimpanzee. Immunogenetics 47 212-217. (doi:10.1007/ s002510050350)

Enders AC \& Blankenship TN 1997 Modification of endometrial arteries during invasion by cytotrophoblast cells in the pregnant macaque. Acta Anatomica 159 169-193. (doi:10.1159/000147983) 
Enders AC, Lantz KC \& Schlafke S 1996 Preference of invasive cytotrophoblast for maternal vessels in early implantation in the macaque. Acta Anatomica 155 145-162. (doi:10.1159/000147800)

Garovic VD, Wagner SJ, Turner ST, Rosenthal DW, Watson WJ, Brost BC, Rose CH, Gavrilova L, Craigo P, Bailey KR et al. 2007 Urinary podocyte excretion as a marker for preeclampsia. American Journal of Obstetrics and Gynecology 196 320.e1-320.e7. (doi:10.1016/j.ajog.2007.02.007)

Harris LK 2011 IFPA Gabor Than Award lecture: Transformation of the spiral arteries in human pregnancy: key events in the remodelling timeline. Placenta [in press]. (doi:10.1016/j.placenta.2010.11.018)

Hiby SE, Walker JJ, O'Shaughnessy KM, Redman CW, Carrington M, Trowsdale J \& Moffett A 2004 Combinations of maternal KIR and fetal HLA-C genes influence the risk of preeclampsia and reproductive success. Journal of Experimental Medicine 200 957-965. (doi:10.1084/ jem.20041214)

Hiby SE, Regan L, Lo W, Farrell L, Carrington M \& Moffett A 2008 Association of maternal killer-cell immunoglobulin-like receptors and parental HLA-C genotypes with recurrent miscarriage. Human Reproduction 23 972-976. (doi:10.1093/humrep/den011)

Hiby SE, Apps R, Sharkey AM, Farrell LE, Gardner L, Mulder A, Claas FH, Walker JJ, Redman CC, Morgan L et al. 2010 Maternal activating KIRs protect against human reproductive failure mediated by fetal HLA-C2. Journal of Clinical Investigation 120 4102-4110. (doi:10.1172/ JCl43998)

Hunt JS, Petroff MG, McIntire RH \& Ober C 2005 HLA-G and immune tolerance in pregnancy. FASEB Journal 19 681-693. (doi:10.1096/fj.042078rev)

Ilekis JV, Reddy UM \& Roberts JM 2007 Preeclampsia - a pressing problem: an executive summary of a National Institute of Child Health and Human Development Workshop. Reproductive Sciences 14 508-523. (doi:10. 1177/1933719107306232)

Lash GE, Robson SC \& Bulmer JN 2010 Review: functional role of uterine natural killer (uNK) cells in human early pregnancy decidua. Placenta 31 (Supplement) S87-S92. (doi:10.1016/j.placenta.2009.12.022)

Lawlor DA, Warren E, Taylor P \& Parham P 1991 Gorilla class I major histocompatibility complex alleles: comparison to human and chimpanzee class I. Journal of Experimental Medicine 174 1491-1509. (doi:10.1084/jem.174.6.1491)

Ledford H 2010 Chimps' fate ignites debate: decision to relocate colony of ageing research chimpanzees becomes political. Nature 467 507-508. (doi:10.1038/467507a)

Martin RD 2007 The evolution of human reproduction: a primatological perspective. American Journal of Physical Anthropology 134 (Supplement 45) 59-84. (doi:10.1002/ajpa.20734)

Moesta AK, Abi-Rached L, Norman PJ \& Parham P 2009 Chimpanzees use more varied receptors and ligands than humans for inhibitory killer cell Ig-like receptor recognition of the $\mathrm{MHC}-\mathrm{C} 1$ and $\mathrm{MHC}-\mathrm{C} 2$ epitopes. Journal of Immunology 182 3628-3637. (doi:10.4049/jimmunol. 0803401)

Moesta AK, Graef T, Abi-Rached L, Older Aguilar AM, Guethlein LA \& Parham P 2010 Humans differ from other hominids in lacking an activating NK cell receptor that recognizes the $\mathrm{C} 1$ epitope of $\mathrm{MHC}$ class I. Journal of Immunology 185 4233-4237. (doi:10.4049/jimmunol. 1001951)

Moffett A \& Hiby SE 2007 How does the maternal immune system contribute to the development of pre-eclampsia? Placenta 28 (Supplement A) S51-S56. (doi:10.1016/j.placenta.2006.11.008)

Older Aguilar AM, Guethlein LA, Adams EJ, Abi-Rached L, Moesta AK \& Parham P 2010 Coevolution of killer cell Ig-like receptors with HLA-C to become the major variable regulators of human NK cells. Journal of Immunology 185 4238-4251. (doi:10.4049/jimmunol.1001494)

Pijnenborg R, D'Hooghe T, Vercruysse L \& Bambra C 1996 Evolution of trophoblast invasion in placental bed biopsies of the baboon, with immunohistochemical localisation of cytokeratin, fibronectin, and laminin. Journal of Medical Primatology 25 272-281.

Pijnenborg R, Vercruysse L \& Hanssens M 2006 The uterine spiral arteries in human pregnancy: facts and controversies. Placenta 27 939-958. (doi:10.1016/j.placenta.2005.12.006)

Pijnenborg R, Vercruysse L \& Carter AM 2010 Trophoblast invasion and uterine artery remodelling in primates. In The Placenta and Human Developmental Programming, pp 92-101. Eds GJ Burton, DJP Barker, A Moffett \& K Thornburg. Cambridge, UK: Cambridge University Press.

Ramsey EM, Houston ML \& Harris JWS 1976 Interactions of the trophoblast and maternal tissues in three closely related primate species. American Journal of Obstetrics and Gynecology 124 647-652.

Roberts JM \& Lain KY 2002 Recent insights into the pathogenesis of preeclampsia. Placenta 23 359-372. (doi:10.1053/plac.2002.0819)

Robillard PY, Dekker GA \& Hulsey TC 2002 Evolutionary adaptations to preeclampsia/eclampsia in humans: low fecundability rate, loss of oestrus, prohibitions of incest and systematic polyandry. American Journal of Reproductive Immunology 47 104-111. (doi:10.1034/j.16000897.2002.10043.x)

Slukvin II, Watkins DI \& Golos TG 2001 Phenotypic and functional characterization of rhesus monkey decidual lymphocytes: rhesus decidual large granular lymphocytes express CD56 and have cytolytic activity. Journal of Reproductive Immunology 50 57-79. (doi:10.1016/ S0165-0378(00)00090-5)

Stout C \& Lemon WB 1969 Glomerular capillary swelling in a pregnant chimpanzee. American Journal of Obstetrics and Gynecology 105 $212-215$.

Thornton JG \& Onwude JL 1992 Convulsions in pregnancy in related gorillas. American Journal of Obstetrics and Gynecology 167 240-241.

Varki A \& Altheide TK 2005 Comparing the human and chimpanzee genomes: searching for needles in a haystack. Genome Research 15 1746-1758. (doi:10.1101/gr.3737405)

Received 21 December 2010

First decision 17 January 2011

Accepted 27 January 2011 